\title{
Quantum Properties of Two-mode Squeezed Thermalized States
}

\author{
Mohammed Kelif \\ Department of physics, Kebridar University, Kebridar, Ethiopia \\ Alemayehu Getahun \\ Department of physics, Mekdela Amba University, Tullu Awulia, Ethiopia
}

\begin{abstract}
We have studied the statistical and squeezing properties of the Two-mode squeezed thermal light. We have calculated the mean of the photon number sum and difference, the variance of the photon number sum and difference, second order correlation function, the quadrature variance and quadrature squeezing, employing the $\mathrm{Q}$ function. We have found that the mean of the photon number sum for the two-mode squeezed thermal light is the sum of the means of the photon number sum of the separate modes and the photon statistic is super Poissonian. And the photon number of a two-mode squeezed thermal light is correlated and we have seen that the two-mode squeezed thermal light exhibiting the photon bunching effect. Moreover, the quadrature variance of the two-mode squeezed thermal light turns out to be the product of the quadrature variances of the two-mode thermal and twomode squeezed vacuum states. The squeezing increase with increasing the squeeze parameter and with decreasing the mean photon number of thermal light.
\end{abstract}

DOI: $10.7176 / \mathrm{JNSR} / 11-23-01$

Publication date: December $31^{\text {st }} 2020$

\section{Introduction}

Nowadays, quantum optics, the union of quantum field theory and physical optics, is undergoing a time of revolutionary change. The subject has evolved from early studies on the coherence properties of radiation to the laser in the modern areas of study involving, e.g. the role of squeezed states of the radiation field and atomic coherence in quenching quantum noise in interferometry and optical amplifiers [1],[2]. For the description of a quantum system, the concept of state is used, which is the same, a wave function, a state vector or a density matrix, quasi-probability distribution containing the information about the possible results of measurement on the system. Furthermore, quantum optics has statistical origin and therefore the state of a quantum system contains all information necessary to completely determine its statistics (the probabilistic nature of a quantum system) [3]-[10]. In this paper, we seek to study the statistical and squeezing properties of two-mode squeezed chaotic light. First, we obtain the anti- normally ordered characteristic function and with the aid of which we obtain the Q-function. To this end, applying the resulting Q-function, we calculate the mean of the photon number sum and difference, the variance of the photon number sum and difference and using the mean of the separate modes a long with the expectation value of $\hat{n}_{\mathrm{a}} \hat{n}_{\mathrm{b}}$ we calculate the second order correlation function. Finally, we calculate the quadrature variances and quadrature squeezing for signal-idler modes with aid of Q-function.

\section{Thermalized two-mode Light}

Thermal light is a typical example of a light mode in a chaotic state. This is generated by a source in thermal equilibrium at some temperature. We can describe it interims of the single-mode density operator as;

$$
\hat{\rho}_{1 T}=\sum_{n_{1}=0}^{\infty} \frac{\bar{n}_{1} n_{1}}{\left(1+\bar{n}_{1}\right)^{n_{1}+1}}\left|n_{1}\right\rangle\left\langle n_{1}\right|
$$

The density operator for two mode chaotic light can be treated as the product of the density operator for two single mode chaotic light [11]

$$
\hat{\rho}_{T}=\hat{\rho}_{1 T} \otimes \hat{\rho}_{2 T}
$$

Using Eq. (1) into Eq. (2), we have

$$
\hat{\rho}_{T}=\sum_{n=0}^{\infty} \frac{\bar{n}_{1}^{n_{1}}{\overline{n_{2}}}^{n_{2}}}{\left(1+\bar{n}_{1}\right)^{n_{1}+1}\left(1+\bar{n}_{1}\right)^{n_{2}+1}}\left|n_{1}, n_{2}\right\rangle\left\langle n_{1}, n_{2}\right|
$$

\subsection{Two-mode squeezed states.}

A nondegenerate sub harmonic system is a typical source of a two-mode squeezed light. In this system a pump 
photon of frequency $\omega c$ is down converted into highly correlated signal and idler photons of frequency $\omega \mathrm{a}$ and $\omega \mathrm{b}$ such that $\omega \mathrm{c}=\omega \mathrm{a}+\omega \mathrm{b}$. A nondegenerate sub harmonic system is described by the Hamiltonian [13].

$$
H=i \epsilon\left(\hat{a} \hat{b}-\hat{a}^{\dagger} \hat{b}^{\dagger}\right)
$$

On account of Eq. (4) we can set the two-mode squeezing operator as;

$$
\hat{S}(r)=e^{r\left(\hat{a} \hat{b}-\hat{a}^{\dagger} \hat{b}^{\dagger}\right)}
$$

To this end, introducing the operator $\hat{a}(\mathrm{r})$ and $\hat{b}(\mathrm{r})$ respectively as [11].

$$
\hat{a}(r)=\hat{S}^{\dagger}(r) \hat{a} \hat{S}(r) \text { and } \hat{b}(r)=\hat{S}^{\dagger}(r) \hat{b} \hat{S}(r)
$$

Differentiating both of the above equation wrt $r$ and using trigonometric identity we get

$$
\hat{a}(r)=\hat{a} \cosh r-\hat{b}^{\dagger} \sinh r
$$

And

$$
\hat{b}(r)=\hat{b} \cosh r-\hat{a}^{\dagger} \sinh r
$$

With help of Eq. (3) and (5) the density operator for the two-mode squeezed chaotic state takes the form

$$
\hat{\rho}=\hat{S}(r) \hat{\rho}_{T} \hat{S}^{\dagger}(r)
$$

\section{The $\mathbf{Q}$ function}

Here we confine our discussion to the $\mathrm{Q}$ function which is best suited to the evaluation of the expectation value of anti -normally ordered operators [3]- [12].

$$
Q(\alpha, \beta, r)=\frac{1}{\pi^{4}} \int d^{2} z d^{2} \eta \phi_{a}(z, \eta, r) \exp \left(z^{*} \alpha-\alpha^{*} z+\eta \beta^{*}-\beta \eta^{*}\right)
$$

Where

$$
\begin{aligned}
\phi_{a}(z, \eta, r) & =\frac{1}{\bar{n}_{a} \bar{n}_{b}} \int \frac{d^{2} \alpha d^{2} \beta}{\pi^{2}} \exp \left[\left(-\frac{\alpha \alpha^{*}}{\bar{n}_{a}}-\frac{\beta \beta^{*}}{\bar{n}_{b}}\right)\right] \\
& \times \exp \left[\left(z \cosh r+\eta^{*} \sinh r\right) \alpha^{*}-\left(z^{*} \cosh r+\eta \sinh r\right) \alpha\right] \\
& \times \exp \left(\left(\eta \cosh r+z^{*} \sinh r\right) \beta^{*}-\left(\eta^{*} \cosh r+z \sinh r\right) \beta\right)
\end{aligned}
$$

Applying integration both wrt $\beta$ and $\alpha$ respectively using

$$
\int \frac{d^{2} z}{\pi} \exp \left(-a z z^{*}+b z+c z^{*}\right)=\frac{1}{a} e^{\frac{b c}{a}}, a>0
$$

We find

$$
\phi_{a}(z, \eta, r)=\exp \left[-a z z^{*}-\eta \eta^{*} b-c\left(z \eta+\eta^{*} z^{*}\right)\right]
$$

Where

$$
\begin{gathered}
a=\left(1+\bar{n}_{1}\right) \cosh ^{2} r+\bar{n}_{2} \sinh ^{2} r \\
b=\left(1+\bar{n}_{2}\right) \cosh ^{2} r+\bar{n}_{1} \sinh ^{2} r
\end{gathered}
$$

And

$$
c=\left(1+\bar{n}_{1}+\bar{n}_{2}\right) \sinh r \cosh r
$$

Using Eq. (13) into Eq. (10) we can set the Q function in the form of 


$$
Q=\frac{A}{\pi^{2}} \exp \left[-u \alpha \alpha^{*}-v \beta \beta^{*}-r\left(\alpha \beta+\alpha^{*} \beta^{*}\right)\right]
$$

In which

$$
A=u v-r^{2}=\frac{1}{a b-c^{2}} \quad, \quad u=\frac{b}{a b-c^{2}}, \quad v=\frac{a}{a b-c^{2}} \quad r=\frac{c}{a b-c^{2}} .
$$

\section{Photon Statistics}

The statistical properties of a light are described in terms of the mean and variance of the photon number. In this section applying the Q-function, we seek to determine the mean and variance of the photon numbers sum and difference for mode a and mode $b$.

\subsection{The mean of the photon number sum and difference}

Here, employing the Q-function, we proceed to study the quantum and statistical properties of the two-mode squeezed chaotic light. The mean of the photon numbers sum and difference can be expressed in terms of Qfunction as [3].

$$
\bar{n}_{ \pm}=\bar{n}_{a} \pm \bar{n}_{b}=\int d^{2} \alpha Q\left(\alpha, \alpha^{*}, r\right) n_{a}(\alpha) \pm \int d^{2} \beta Q\left(\beta, \beta^{*}, r\right) n_{b}(\beta)
$$

Substituting Eq. (17) into Eq. (18) And applying integration with help of Eq.(12) the mean of the photon number sum and difference can putted as;

$$
\bar{n}_{ \pm}=(1 \pm 1) \sinh ^{2} r+\bar{n}_{1} \pm \bar{n}_{2}
$$

From this equation, we have

$$
\bar{n}_{+}=2 \sinh ^{2} r+\bar{n}_{1}+\bar{n}_{2}
$$

And

$$
\bar{n}_{-}=\bar{n}_{1}-\bar{n}_{2}
$$

From Fig. (1) We observe that the mean photon number of the two-mode squeezed chaotic light is the sum of a two-mode squeezed and a two-mode chaotic light. But, difference mean photon number is equal to the mean photon number difference of two mode chaotic light. Thus, indicate that the mean photon number of two-mode squeezed lights is equal.

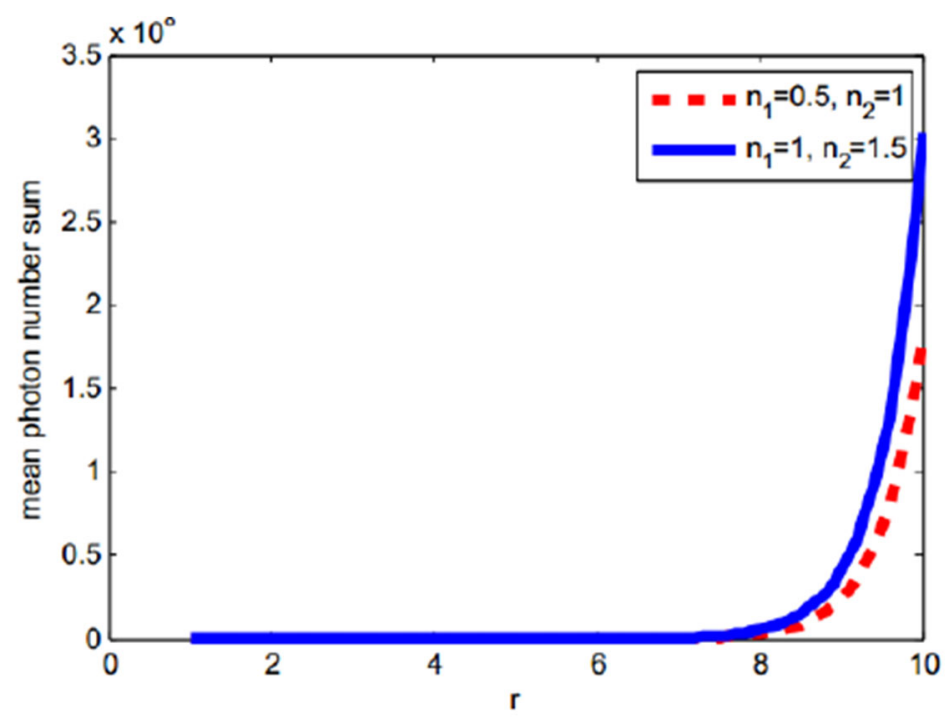

Figure 1: plot of the mean of photon number sum [Eq. (19)] versus squeeze parameter $r$ and For different values of $\bar{n}_{1}$ and $\bar{n}_{2}$.

\subsection{The variance of the photon number sum and difference}

We now proceed to determine the variance of the photon number sum and difference for the two-mode squeezed chaotic (thermal) light. For a two-mode light, the variance of the photon number sum and difference is expressible 
in the form [4].

$$
\left(\Delta n_{ \pm}\right)^{2}=\Delta n_{a}^{2}+\Delta n_{b}^{2} \pm 2\left[\left\langle\hat{n}_{a} \hat{n}_{b}\right\rangle-\bar{n}_{a} \bar{n}_{b}\right]
$$

Using several mathematical formulas we can set

$$
\begin{aligned}
\Delta n_{a}^{2} & =\bar{n}_{1}^{2} \cosh ^{4} r+\bar{n}_{1} \cosh ^{2} r-\bar{n}_{2} \sinh ^{2} r+\bar{n}_{2}^{2} \sinh ^{4} r \\
& +\left(2 \bar{n}_{1} \bar{n}_{2}+2 \bar{n}_{2}+2 \bar{n}_{1}+1\right) \cosh ^{2} r \sinh ^{2} r \\
\Delta n_{b}^{2} & =\bar{n}_{2}^{2} \cosh ^{4} r+\bar{n}_{2} \cosh ^{2} r-\bar{n}_{1} \sinh ^{2} r+\bar{n}_{1}^{2} \sinh ^{4} r \\
& +\left(2 \bar{n}_{1}+2 \bar{n}_{1} \bar{n}_{2}+2 \bar{n}_{2}+1\right) \cosh ^{2} r \sinh ^{2} r
\end{aligned}
$$

And

$$
\begin{aligned}
\left\langle\hat{n}_{a} \hat{n}_{b}\right\rangle & =1+\left(\bar{n}_{1}+\bar{n}_{2}+\bar{n}_{1} \bar{n}_{2}+1\right) \cosh ^{4} r-\bar{n}_{1} \bar{n}_{2} \sinh ^{2} r \\
& +\left[1+3\left(\bar{n}_{1}+\bar{n}_{2}+\bar{n}_{1} \bar{n}_{2}\right)+2\left(\bar{n}_{1}^{2}+\bar{n}_{2}^{2}\right)\right] \cosh ^{2} r \sinh ^{2} r \\
& -\left(\left(1+\bar{n}_{2}\right) \cosh ^{2} r+\left(\bar{n}_{1}+\bar{n}_{2}\right) \sinh ^{2} r+\left(1+\bar{n}_{1}\right) \cosh ^{2} r\right) \\
\bar{n}_{a} \bar{n}_{b} & =\left(1+2 \bar{n}_{1}+2 \bar{n}_{2}+\bar{n}_{1}^{2}+\bar{n}_{2}^{2}+2 \bar{n}_{1} \bar{n}_{2}\right) \cosh ^{2} r \sinh ^{2} r \\
& +\bar{n}_{1} \bar{n}_{2}-\left(1+\bar{n}_{1}+\bar{n}_{2}\right) \sinh ^{2} r
\end{aligned}
$$

Using Eqs. (23)- (26) into Eq. (22) the variance photon number sum and difference for two-mode thermalized squeezed vacuum states takes the form;

$$
\begin{aligned}
\left(\Delta n_{ \pm}\right)^{2} & =(1 \pm 1)\left(2+4 \bar{n}_{1} \bar{n}_{2}+4 \bar{n}_{1}+4 \bar{n}_{2}+2 \bar{n}_{1}^{2}+2 \bar{n}_{2}^{2}\right) \cosh ^{2} r \sinh ^{2} r \\
& +\bar{n}_{1}+\bar{n}_{2}+\bar{n}_{1}^{2}+\bar{n}_{2}^{2}
\end{aligned}
$$

From this equation we can simply states the variance photon number sum and difference respectively as;

$$
\left(\Delta n_{+}\right)^{2}=4\left(1+2 \bar{n}_{1}\right)^{2} \cosh ^{2} r \sinh ^{2} r+2 \bar{n}_{1}\left(1+\bar{n}_{1}\right)
$$

And

$$
\left(\Delta n_{-}\right)^{2}=\bar{n}_{1}+\bar{n}_{2}+\bar{n}_{1}^{2}+\bar{n}_{2}^{2}
$$

From Eqs. (20), (21), (28) and Eq. (29) we can understand that the sum and difference variance of photon number is greater than that of mean photon number which indicates the system gas superpoissonian photon statistics.

\subsection{The second order correlation function}

In this section, we seek to determine the normalized photon numbers correlation and either can exhibit photon bunching or anti-bunching effect using the mean of the separate modes a long with the expectation value of $\bar{n}_{a} \bar{n}_{b}$ for the system under consideration.

\subsubsection{The normalized photon number correlation}

In order to determine whether the photon numbers of mode $a$ and mode $b$ are correlated or not, we must examine the normalized photon numbers correlation. Thus the photon numbers correlation of the two-mode light can be defined by [1],[3], [8]

$g_{a b}^{(2)}(r)=\frac{\left\langle\hat{n}_{a} \hat{n}_{b}\right\rangle}{\left\langle\hat{n}_{a}\right\rangle\left\langle\hat{n}_{b}\right\rangle}$

Where 


$$
\left\langle\hat{n}_{a} \hat{n}_{b}\right\rangle=\left\langle\hat{n}_{a}\right\rangle\left\langle\hat{n}_{b}\right\rangle+\left(1+2 \bar{n}_{1}+2 \bar{n}_{2}+2 \bar{n}_{1} \bar{n}_{2}+\bar{n}_{1}^{2}+\bar{n}_{2}^{2}\right) \cosh ^{2} r \sinh ^{2} r
$$

On account of Eq. (31), Eq. (30) takes the form

$g_{a b}^{(2)}(r)=1+\frac{\left(1+2 \bar{n}_{1}+2 \bar{n}_{2}+2 \bar{n}_{1} \bar{n}_{2}+\bar{n}_{1}^{2}+\bar{n}_{2}^{2}\right) \cosh ^{2} r \sinh ^{2} r}{\left\langle\hat{n}_{a}\right\rangle\left\langle\hat{n}_{b}\right\rangle}$

In particular, in the absence of thermal light $\left(\bar{n}_{1}=\bar{n}_{2}=0\right)$, Eq. (32) becomes

$$
g_{a b}^{(2)}(r)=1+\operatorname{coth}^{2} r
$$

These results indicates that the photon numbers correlation is different from one. Thus the photon numbers of mode a and mode b of a two-mode Squeezed Chaotic light and the two-mode squeezed vacuum states are correlated. And we see that the Chaotic light has no effect on the photon numbers correlation.

\subsubsection{Photon anti-bunching}

We seek to determine whether the two-mode squeezed thermal light is either can exhibit the photon bunching or anti-bunching effect. The criterion for the existence of the anti-bunching effect in the two-mode radiation field is given by [7].

Where

$$
R_{a b}^{(2)}(r)=\frac{\left\langle\hat{a}^{\dagger 2} \hat{a}^{2}\right\rangle+\left\langle\hat{b}^{\dagger 2} \hat{b}^{2}\right\rangle}{2\left\langle\hat{a}^{\dagger} \hat{a} \hat{b}^{\dagger} \hat{b}\right\rangle}-1<0
$$

$$
\left\langle\hat{a}^{\dagger 2} \hat{a}^{2}\right\rangle=2\left[\left(1+2 \bar{n}_{1}+2 \bar{n}_{2}+2 \bar{n}_{1} \bar{n}_{2}+\bar{n}_{1}^{2}+\bar{n}_{2}^{2}\right) \sinh ^{2} r \cosh ^{2} r\right.
$$

$$
\left.-\left(\bar{n}_{2}^{2}+2+2 \bar{n}_{2}\right) \sinh ^{2} r+2\left(1+\bar{n}_{1}^{2}\right) \cosh ^{2} r-1\right]
$$

And

$$
\begin{gathered}
\left\langle\hat{b}^{\dagger 2} \hat{b}^{2}\right\rangle=2\left[\left(1+2 \bar{n}_{1}+2 \bar{n}_{2}+2 \bar{n}_{1} \bar{n}_{2}+\bar{n}_{1}^{2}+\bar{n}_{2}^{2}\right) \sinh ^{2} r \cosh ^{2} r\right. \\
\left.-\left(\bar{n}_{1}^{2}+2+2 \bar{n}_{1}\right) \sinh ^{2} r+2\left(1+\bar{n}_{2}^{2}\right) \cosh ^{2} r-1\right]
\end{gathered}
$$

Substituting Eqs. (36) and (35) into Eq. (34) we find

$$
R_{a b}^{(2)}(r)=\frac{\bar{n}_{1}^{2}+\bar{n}_{2}^{2}-\left(1+\bar{n}_{1}+\bar{n}_{2}\right) \sinh ^{2} r-\bar{n}_{1} \bar{n}_{2}}{\left\langle\hat{n}_{a} \hat{n}_{b}\right\rangle}
$$

In particular, when $\bar{n}_{1}=\bar{n}_{2}=0$, Eq. (37) becomes

$$
R_{a b}^{(2)}(r)=\frac{-1}{2 \cosh ^{2} r-1}<0
$$

From this result, we observe that the two mode squeezed vacuum state is anti-bunching.

\subsection{The quadrature squeezing}

In this section, we seek to determine the variance and squeezing of the quadrature operator for the system under consideration with the aid of Q-function.

\subsubsection{The quadrature variances}

The calculation of the variances in a quantum state leads to the determination of the total noise of that state. The variances in a two-mode state are defined as the mean square uncertainties in the real and imaginary parts of the 
annihilation operator of the mode. Hence the total noise of the state is given by the sum of the variances in that state [4],[12].

$$
\left(\Delta c_{ \pm}\right)^{2}=2+\left\langle: \hat{c}_{ \pm}, \hat{c}_{ \pm}:\right\rangle
$$

After calculating both normal order quadrature operator we can set the plus and minus quadrature as;

$$
\begin{aligned}
\left(\Delta c_{ \pm}\right)^{2} & =2\left(2+\bar{n}_{1}+\bar{n}_{2}\right) \sinh ^{2} r+2\left(\bar{n}_{1}+\bar{n}_{2}\right) \cosh ^{2} r \\
& \pm 4\left(1+\bar{n}_{1}+\bar{n}_{2}\right) \sinh r \cosh r+2
\end{aligned}
$$

In which the plus and minus quadrature's are given by

$$
\left(\Delta c_{+}\right)^{2}=2\left(1+n_{T}\right) e^{-2 r}
$$

And

$$
\left(\Delta c_{-}\right)^{2}=2\left(1+n_{T}\right) e^{2 r}
$$

Fig.2 represent the plot of quadrature variance [Eq. (41)] versus $r$ and for different values of $n_{T}$. From the graph we realize that the quadrature variance increases with increasing $\mathrm{n}_{T}$ and decreases with increasing squeeze parameter $\mathrm{r}$.

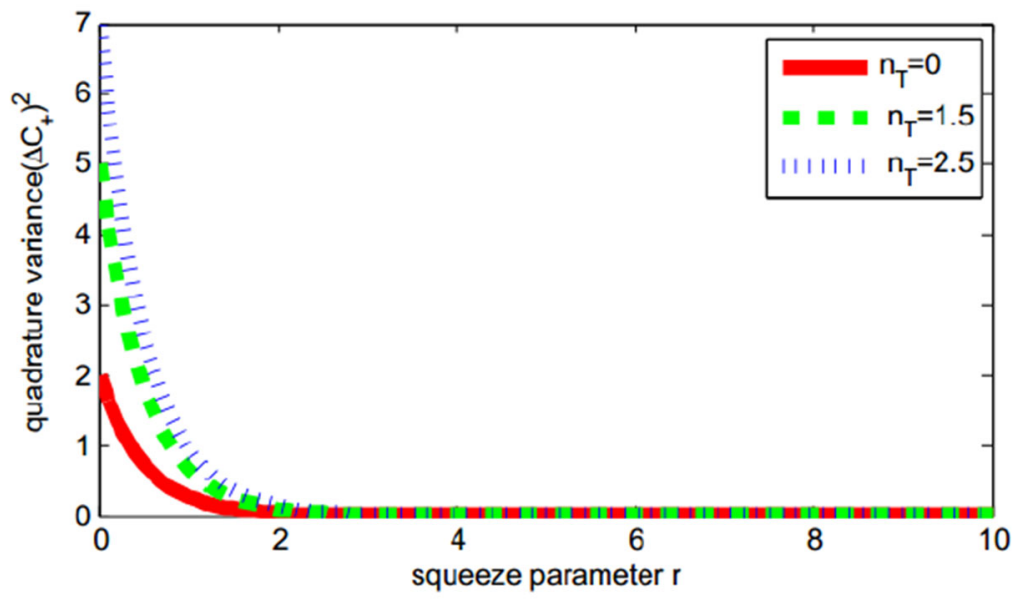

Figure 2: plot of quadrature variance [Eq. (41)] versus $r$ and for different values of $n_{T}$.

\subsubsection{Quadrature squeezing}

A two mode light is said to be in a squeezed state if $\Delta \mathrm{c}_{+}<2$ or $\Delta \mathrm{c}_{-}<2$ such that $\Delta \mathrm{c}_{+} \Delta \mathrm{c}_{-} \geq 2$. Thus, the quadrature squeezing of the two-mode light can be defined in terms of the quadrature variance of a two mode coherent light as;

$$
S_{+}=\frac{\left(\Delta c_{+}\right)_{c}^{2}-\left(\Delta c_{+}\right)^{2}}{\left(\Delta c_{+}\right)_{c}^{2}}
$$

Where

$$
\left(\Delta c_{+}\right)_{c}^{2}=2
$$

On account Eqs. (41) and (44), Eq. (43) takes the form

$$
S_{+}=1-\left(1+n_{T}\right) e^{-2 r}
$$

Eq. (45) is called as the plus quadrature squeezing 


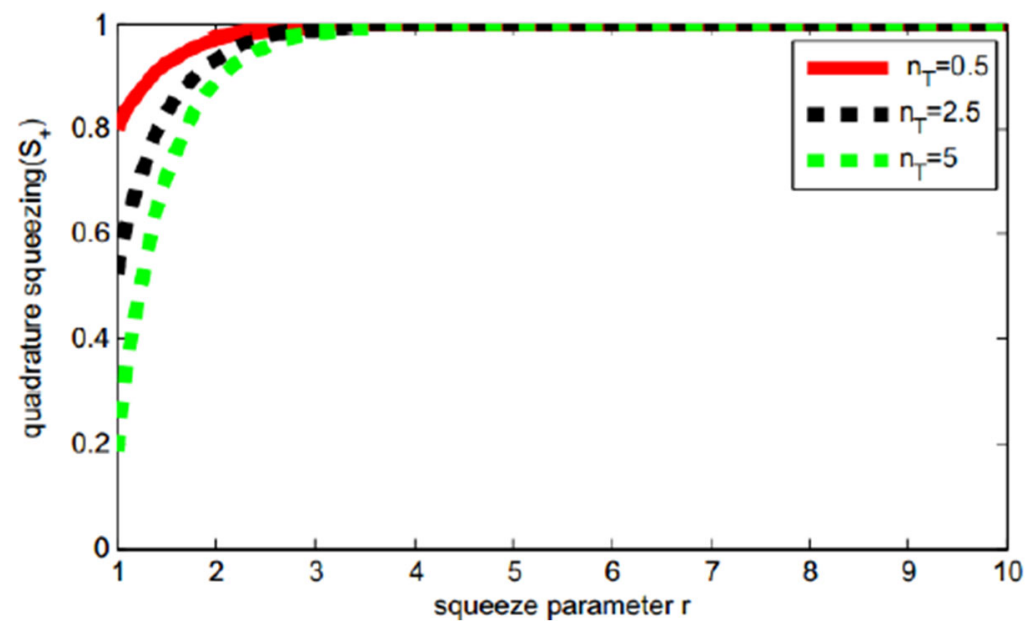

Figuare 3: plot of quadrature squeezing of Eq. (45) versus squeeze parameter $r$ and for different Values of $n_{T}$

Plot 3 represent quadrature squeezing of Eq. (45) versus squeeze parameter $r$ and for different values of $n_{T}$. From this figure we see that quadrature squeezing increase as squeeze parameter $r$ increase, $n_{T}$ is decreases. The graph also show that the degree of squeezing decrease as the value of $\mathrm{n}_{\mathrm{T}}$ is increases.

\section{Conclusions}

In this paper, we studied the statistical and squeezing properties of two-mode squeezed thermal light. First, we obtained the anti-normally-ordered characteristic function, following the procedure discussed in [13] and with the aid of which we obtained the Q-function.

Applying the resulting Q-function, we have calculated the mean of the photon number sum and difference, the variance of the photon number sum and difference and using the mean of the separate modes a long with the expectation value of ^nânb, we calculated the second order correlation function. Finally, we calculated the quadrature variances and quadrature squeezing for signal-idler modes with aid of Q-function. The result shows that the statistical and squeezing properties of two mode lights are affected by a two mode thermal light. The effect of two mode thermal light is to increase mean of the photon number sum and variance of the photon number sum and difference and decrease the squeezing properties of two mode light and we found that the mean of the photon number sum for the two-mode squeezed chaotic light is the sum of the mean photon number of the separate modes and the mean of the photon number difference is zero. The variance of the photon number sum and difference (equal to the variance of the photon number difference of the two mode chaotic light) for the two mode squeezed chaotic light is greater than the mean photon number sum and difference. Thus the photon statistics of a two-mode squeezed thermal light is super poissonian. The photon number of a two-mode squeezed thermal lights are correlated and we have seen that the two-mode squeezed thermal light exhibiting the bunching effect, thus a twomode squeezed thermal light is classic.

Moreover, the quadrature variance of the two-mode squeezed chaotic light is the product of the quadrature variance of the two mode squeezed vacuum and the quadrature variance of the two mode chaotic light, and the fluctuation in the plus and minus quadrature variances are dependent on the mean photon number of thermal light and squeeze parameter. For large value of mean photon number of thermal light, they are above the coherent state level and for very small value of the mean photon number of thermal light, squeezing occur in the plus quadrature. The squeezing increase with increasing the squeeze parameter $r$.

\section{Reference}

[1] Faisal Aly El-Orany, Quantum properties of Superpostion states,Squeezed states, PhD Dissertation (Palacky University, 2001).

[2] Mark Pox, introduction quantum optics (University of Sheffield, 2005).

[3] Tizazu Masresha, Superposition of Squeezed Laser Light,PHDDissertation (Addis Ababa University, 2015)

[4] M.O. Scully and, M.S. Zubary, Quantum optics (CambridgeUniversity press Cambridge1997).

[5] A. I.Lvovsky, Squeezed light (Institute for Quantum Information Science, University of Calgary, 2014).

[6] FAN et al, Chin. Phys. Lett. vol. 28, No.4(2011) 040302.

[7] Wang et al, Chin.phys. B vol.23,No.2(2014) 024206.

[8] Mesfin Ayele, Two mode coherent and squeezed light, Msc.thesis(Addis ababa University, 2012)

[9] Wan et al, Chin. phys. B vol.24 No.12(2015) 120301.

[10] Kassahun, Fundamentals of quantum optics (Lulu Press Inc. North Carolina, 2010). 
[11] Assamen Ayalew and Fesseha kassahun, Superposed Two mode chaotic, coherent, and Squeezed Vacuum State (Aksum University and Addis AbabaUniversity, July 3,2014).

[12] Fesseha Kassahun, arXiv: 1201, 3712v1 [quant-phys] (2012).

[13] Fesseha Kassahun, Fundamentals of Quantum Optics (Lulu, North carolina, 2008).

[14] W.H. Louis ell, Quantum stastical properties of radiation (New York, 1973). 